\title{
Aneurysm formation and bradykinin
}

\author{
Friedrich C. Luft
}

Published online: 26 August 2009

(C) Springer-Verlag 2009

In this issue, Merino et al. publish a provocative paper on accelerated aortic aneurysm formation in mice lacking both apolipoprotein E (APOE) and the bradykinin[1] (B1) receptor [1]. Their disease model is the APOE genedeleted (-/-) mouse that I admittedly love. These are indeed the mice that "keep on giving" [2]. APOE-/- mice not only are cooperative in terms of developing atherosclerosis but also develop aortic aneurysms when infused with angiotensin (Ang) II, making them an ideal model for studying this serious clinical disease [3]. APOE-/- mice are not unique in this regard; low-density lipoprotein receptor $-/-$ mice work equally as well. The role of Ang II is interesting and is independent of the blood pressure increase [4]. Norepinephrine, on the other hand, is much less prone to provoke aneurysm formation.

Merino et al. [1] fed APOE gene-deficient mice and APOE-deficient mice that had been crossed into a B1 receptor -/- model namely, an APOE-/- and B1-/"double-knockout" model, a high-fat diet. The cholesterol elevations of both groups were the same. However, the absent B1 receptors made the atherosclerosis and aneurysm formation worse. The expressions of cyclic guanylyl monophosphate (cGMP)-dependent kinase I, CD-11, F4/ 80 , monocyte colony-stimulating factor (mCSF), and tumor necrosis factor (TNF)- $\alpha$ were also increased in the aortas of the double knockout, compared to APOE-/- mice. Peroxisome proliferator-activated receptor $\gamma(\operatorname{PPAR} \gamma)$ protein and the matrix metalloproteinase-9 (MMP9) activity, on the other hand, were decreased in the aortas of the doubleknockout mice. Remarkably, Ang II was not required for aneurysm formation, although Ang II infusion experiments

F. C. Luft $(\bowtie)$

Experimental and Clinical Research Center,

Berlin, Germany

e-mail: luft@charite.de in the mice were conducted. Again, aneurysm formation in the double-knockout mice was worse than in other groups.

What is it about the bradykinin B1 receptor? The B1 receptor plays an important role in mediating the inflammatory effects of the kallikrein-kinin pathway. The B1 receptor $-/-$ mice have revealed that the receptor plays a pivotal role in the cellular, particularly neutrophil, recruitment associated with acute inflammatory responses. Neutrophil recruitment has been implicated in the early stages of atherosclerosis. The B1 receptor plays a major role in chemokine (CXCL5 and CCL2) expression. Thus, we might have speculated that absence of the B1 receptor would protect from atherosclerosis and aneurysm formation, rather than the other way around. The authors argue that upregulation of cGMP-dependent kinase I could have facilitated the atherosclerosis. Indeed, a proatherosclerotic role for cGMP-dependent protein kinase I has been described in vascular smooth muscle cells [5]. The cGMP-dependent protein kinase I is a potential mediator of nitric oxide (NO) signaling in vascular smooth muscle cells. Postnatal ablation of cGMP-dependent protein kinase I selectively in the vascular smooth muscle cells of mice reduced atherosclerotic lesion area, demonstrating that smooth muscle cGMP-dependent protein kinase I promotes atherogenesis. Cell fate mapping in these studies indicated that cGMP-dependent protein kinase I is involved in the development of vascular smooth-muscle-derived plaque cells. Activation of endogenous cGMP-dependent protein kinase I in vascular smooth muscle cells resulted in cells with increased levels of proliferation. Increased levels of vascular cell adhesion molecule-1, peroxisome proliferatoractivated receptor gamma, phosphatidylinositol 3-kinase/ Akt signaling, and decreased plasminogen activator inhibitor $1 \mathrm{mRNA}$ all are potentially proatherogenic properties. The authors suggested that activation of smooth muscle cGMP-dependent protein kinase I contributes to the proatherogenic effect of $\mathrm{NO}$ and that inhibition of cGMP- 
dependent protein kinase I might even be a therapeutic option for treating atherosclerosis in humans.

Merino et al. noted that MMP9 was downregulated in their double-knockout mice [1]. Oddly, patients with atherosclerotic aortic aneurysms have higher circulating MMP9 levels than control subjects, and MMP9 is implicated in aneurysm formation. Suppression of MMP9 has been considered as a treatment for aortic aneurysm [6]. A clinical trial of doxycycline for MMP9 inhibition in patients with an abdominal aneurysm reported that doxycycline selectively depletes aortic wall neutrophils and cytotoxic $\mathrm{T}$ cells [7]. Merino et al. also observed that PPAR $\gamma$ was downregulated in their double-knockout mice [1]. PPAR $\gamma$ is said to inhibit inflammatory cytokines, including interleukin 6 and TNF- $\alpha$. Conceivably, the TNF- $\alpha$ elevation is related to PPAR $\gamma$ downregulation.

Ang II made the tendency for aneurysm formation worse, although double-knockout mice given Ang II faired the poorest of all the groups. Ang II may aggravate aneurysms through upregulation of osteoprotegerin, which has been associated with aneurysm expansion. Others found that APOE-/- mice infused with Ang II had elevated osteoprotegerin in the aortic wall. Ang II increased osteoprotegerin in cultured vascular smooth muscle cells. PPAR $\gamma$ activation decreases osteoprotegerin, as well as MMP9 [8]. Osteoprotegerin, also known as osteoclastogenesis inhibitory factor, is a cytokine, which can inhibit the production of osteoclasts. Osteoprotegerin is a member of the TNF receptor superfamily. It is a basic glycoprotein comprising 401 amino acid residues arranged into seven structural domains. Osteoprotegerin inhibits the differentiation of osteoclast precursors. However, as we see above, osteoprotegerin is a complex protein that evidently also plays a role in vascular signaling and remodeling [9]. Osteoprotegerin is a receptor activator of nuclear factor kappa B (RANK) homolog and works by binding to RANKL ligand on osteoblast and stromal cells, thus blocking the RANKL-RANK ligand interaction between osteoblast/stromal cells and osteoclast precursors. Osteoprotegerin promotes fibrous cap formation in atherosclerotic lesions of APOE-/- mice [10]. Osteoprotegerin also contributes to vascular calcification [11].

Now, how do we justify the entrance of bradykinin into the aneurysm story? Kinins are synthesized from their precursors by different enzymes and participate in the regulation of cardiovascular function through bradykinin B1 and B2 receptors [12]. They modulate blood coagulation by exerting antithrombotic and profibrinolytic actions. By activating B2 receptors that signal process results in the release of nitric oxide and prostacyclin. Kinins inhibit vascular smooth muscle growth and neointima formation, which may play an inhibitory role on the atherosclerosis development. Earlier reports suggested that through the activation of $\mathrm{B} 1$ receptors, kinins could play a deleterious role in this disease. Kinins are potent endogenous vasodilators that are involved in the regulation of coronary vascular tone. However, due to their metabolic characteristics, these peptides act mainly as an autocrine/ paracrine factor to locally regulate blood perfusion of organs. By modulating cellular energy metabolism and myocardial oxygen consumption, kinins protect cardiac and vascular endothelial function in myocardial ischemia and heart failure. Finally, mounting evidence indicates that kinins are involved in the actions of some drugs actually used in the treatment of cardiovascular diseases such as Ang-converting enzyme (ACE) inhibitors and Ang AT1 receptor antagonists (ARB). Thus, the kinin system constitutes a potential therapeutic target for cardiovascular diseases. Experiments in animals attempted to explore the kinin system therapeutically, including

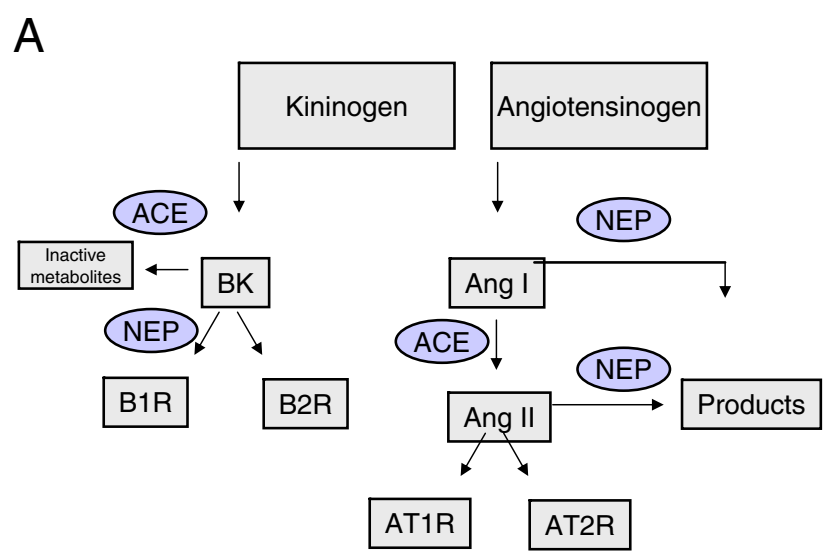

B

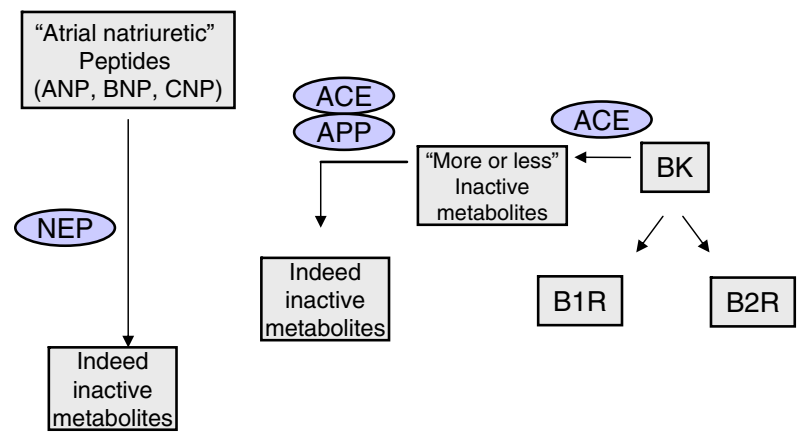

Fig. 1 a shows that angiotensinogen is converted to Ang I (by renin) and that Ang I is converted to Ang II by ACE. This step can be blocked by ACE inhibitors. Ang II addresses two receptors. Ang I and Ang II can both be reduced by NEP to active metabolites not discussed here. Independently, kininogen can be converted to bradykinin (BK), a process mediated by ACE. The products address two receptors, namely $\mathrm{B} 1$ and $\mathrm{B} 2$. b shows that atrial (and brain) natriuretic peptides are cleaved by NEP. These peptides produce natriuresis and lower blood pressure (not discussed here). However, NEP and ACE are integrally interlocked since NEP influences Ang products (a) that are also vasoactive. Finally, NEP (a) has also to do with bradykinin degradation. The issues presented here are a microcosmos of the source [14]. No one said that this would be easy! 
the mobilization of endogenous kinins using pharmacological agents, the application of bradykinin analogs with longacting properties, and the introduction of "gene" therapies. Thus far, there is much promise and little productivity.

Inhibition of bradykinin-metabolizing enzymes can cause acute angioedema, as demonstrated in a recent clinical trial in patients administered the antihypertensive, vasopeptidase inhibitor, omapatrilat [13]. Vasopeptidase inhibitors simultaneously inhibit both ACE and neutral endopeptidase (NEP). These combined effects result in significant lowering of the systemic blood pressure. Vasopeptidase inhibitors are currently not approved because of alleged increased angioedema. However, the relative contribution of specific bradykininmetabolizing enzymes to this effect is unclear and confounded by the lack of a predictive preclinical model of angioedema. Rats were instrumented to record blood pressure and heart rate; inhibitors were infused for $35 \mathrm{~min}$, and bradykinin was infused during the last $5 \mathrm{~min}$ to elicit hypotension, as a functional marker of circulating bradykinin and relative angioedema risk [13]. In the presence of omapatrilat, bradykinin produced dose-dependent hypotension, an effect abolished by B2 receptor blockade. In the presence of lisinopril (ACE inhibitor), but not candoxatril, a NEP inhibitor, or apstatin, an aminopeptidase (APP) inhibitor, bradykinin also elicited hypotension. Lisinopril-mediated hypotension was unchanged with concomitant blockade of NEP or NEP combined with dipeptidase IV (DPPIV) inhibition. However, hypotension was enhanced upon concomitant blockade of APP and further intensified in the presence of NEP inhibition to values not different from omapatrilat alone. Bradykinin is degraded in vivo with an enzyme rank efficacy of ACE $>$ APP $>>$ NEP or DPPIV. This hardly comprehendible pharmacological masterpiece suggests that the effects of omapatrilat are mediated by inhibition of three bradykinin-metabolizing enzymes, ACE/APP/NEP. However, dual inhibition of ACE/NEP or ACE/NEP/DPPIV elicits no increased risk of angioedema compared to ACE inhibition alone. Thus, novel bradykinin-metabolizing enzyme inhibitors must display no activity against APP to avoid angioedema risk due to high prevalence of ACE inhibitor therapy in patients with diabetes and cardiovascular disease.

The authors have the opportunity of performing an interesting pharmacological experiment in their model. APOE-/- mice infused with Ang II develop aneurysms. APOE-/- mice crossed with B1-/- mice develop even more severe aneurysms with Ang II. A vasopeptidase inhibitor, inhibiting both ACE and NEP, should beat an ACE inhibitor in aneurysm protection of $\mathrm{APOE}-/-$ mice. On the other hand, in the double knockouts, ACE inhibitor treatment should be effective, vasopeptidase inhibitor treatment no more effective, and NEP inhibitor treatment alone not effective. A schema, reviewed elsewhere in detail [14], is given in Fig. 1. There are no published papers on the efficacy of omapatrilat on aneurysm formation and progression. Thus far, aneurysm progression prophylaxis with beta-blockers, MMP inhibitors, and similar strategies has been disappointing. In my view, vasopeptidase inhibition to prevent aneurysm progression deserves a look. The APOE-/- and B1-/- model might be just the ticket. Any takers?

Respectfully,

Friedrich C. Luft

\section{References}

1. Merino VJ, Todiras M, Mori MA, Sales VMT, Fonseca RG, Saul V, Tenner K, Bader M, Pesquero JB (2009) Predisposition to atherosclerosis and aortic aneurysms in mice deficient in kinin $\mathrm{B} 1$ receptor and apolipoprotein E. J Mol Med. doi:10.1007/s00109-009-0501-0

2. Luft FC (2000) Love those mice!. J Mol Med 78:179-181

3. Daugherty A, Cassis LA (2004) Mouse models of abdominal aortic aneurysms. Arterioscler Thromb Vasc Biol 24:429-434

4. Cassis LA, Gupte M, Thayer S, Zhang X, Charnigo R, Howatt DA, Rateri DL, Daugherty A (2009) ANG II infusion promotes abdominal aortic aneurysms independent of increased blood pressure in hypercholesterolemic mice. Am J Physiol Heart Circ Physiol 296:H1660-H1665

5. Wolfsgruber W, Feil S, Brummer S, Kuppinger O, Hofmann F, Feil RA (2003) Proatherogenic role for cGMP-dependent protein kinase in vascular smooth muscle cells. Proc Natl Acad Sci U S A 100:13519-13524

6. Takagi H, Manabe H, Kawai N, Goto SN, Umemoto T (2009) Circulating matrix metalloproteinase- 9 concentrations and abdominal aortic aneurysm presence: a meta-analysis. Interact Cardiovasc Thorac Surg. doi:10.1510/icvts.2009.208835

7. Lindeman JH, Abdul-Hussien H, van Bockel JH, Wolterbeek R, Kleemann R (2009) Clinical trial of doxycycline for matrix metalloproteinase- 9 inhibition in patients with an abdominal aneurysm: doxycycline selectively depletes aortic wall neutrophils and cytotoxic T cells. Circulation 119:2209-2216

8. Moran CS, Cullen B, Campbell JH, Golledge J (2009) Interaction between angiotensin II, osteoprotegerin, and peroxisome proliferator-activated receptor-gamma in abdominal aortic aneurysm. J Vasc Res 46:209-217

9. Schoppet M, Preissner KT, Hofbauer LC (2002) RANK ligand and osteoprotegerin: paracrine regulators of bone metabolism and vascular function. Arterioscler Thromb Vasc Biol 22:549-553

10. Ovchinnikova O, Gylfe A, Bailey L, Nordström A, Rudling M, Jung C, Bergström S, Waldenström A, Hansson GK, Nordström P (2009) Osteoprotegerin promotes fibrous cap formation in atherosclerotic lesions of ApoE-deficient mice. Arterioscler Thromb Vasc Biol. doi:10.1161/ATVBAHA.109.188185

11. Orita Y, Yamamoto H, Kohno N, Sugihara M, Honda H, Kawamata S, Mito S, Soe NN, Yoshizumi M (2007) Role of osteoprotegerin in arterial calcification: development of new animal model. Arterioscler Thromb Vasc Biol 27:2058-2064

12. Su JB (2006) Kinins and cardiovascular diseases. Curr Pharm Des 12:3423-3435

13. Fryer RM, Segreti J, Banfor PN, Widomski DL, Backes BJ, Lin CW, Ballaron SJ, Cox BF, Trevillyan JM, Reinhart GA, von Geldern TW (2008) Effect of bradykinin metabolism inhibitors on evoked hypotension in rats: rank efficacy of enzymes associated with bradykinin-mediated angioedema. Br J Pharmacol 153:947-955

14. Daull P, Jeng AY, Battistini B (2007) Towards triple vasopeptidase inhibitors for the treatment of cardiovascular diseases. J Cardiovasc Pharmacol 50:247-256 